PROCEEDINGS OF THE AMERICAN MATHEMATICAL SOCIETY

Volume 124, Number 9, September 1996

\title{
SOME REMARKS ON THE OPERATOR OF FOIAS AND WILLIAMS
}

\author{
SRDJAN PETROVIĆ
}

(Communicated by Palle E. T. Jorgensen)

ABSTRACT. In this paper we study the Foias-Williams operator

$$
T\left(H_{g}\right)=\left(\begin{array}{cc}
S^{*} & H_{g} \\
0 & S
\end{array}\right)
$$

where $g \in L^{\infty}$, and $H_{g}$ is a Hankel operator with symbol $g$. We exhibit a relationship between the similarity of $T\left(H_{g}\right)$ to a contraction and the rate of decay of $\left\{\left|g_{n}\right|\right\}_{n=0}^{\infty}$, the absolute values of the Fourier coefficients of the symbol $g$.

Let $\mathcal{H}$ denote a complex Hilbert space and let $\mathcal{L}(\mathcal{H})$ denote the algebra of all bounded linear operators on $\mathcal{H}$. Recall that an operator $T$ in $\mathcal{L}(\mathcal{H})$ is said to be polynomially bounded if there exists an $M \geq 1$ such that

$$
\|p(T)\| \leq M \sup \{|p(\zeta)|:|\zeta|=1\}
$$

for all polynomials $p$. We denote the class of all polynomially bounded operators by (PB). Also, an operator $T$ is similar to a contraction (notation: $T \in(\mathrm{SC})$ ) if there exists a bounded invertible operator $L$ such that $\left\|L T L^{-1}\right\| \leq 1$. Halmos [4] raised the question whether every polynomially bounded operator is similar to a contraction; this question is still open.

While there is a number of results dealing with sufficient conditions for a polynomially bounded operator to be similar to a contraction (cf. [9], [6], [5]), there are very few publications dedicated to the search for a counterexample. In [3] (see also [2]) Foias and Williams have studied the operators of the form

$$
T(X)=\left(\begin{array}{cc}
S^{*} & X \\
0 & S
\end{array}\right)
$$

acting on $H^{2} \oplus H^{2}$, the direct orthogonal sum of two copies of the Hardy space $H^{2}$ (to be defined below). Here $S$ is a forward unilateral shift on $H^{2}$ and $X \in \mathcal{L}\left(H^{2}\right)$. In particular, they conjectured that there exists a Hankel operator $H_{g}$ with symbol $g$ (to be defined below) such that the operator $T\left(H_{g}\right) \in(\mathrm{PB}) \backslash(\mathrm{SC})$.

In this paper we continue this line of investigation and we show that the membership in the aforementioned classes depends on the rate at which the sequence $\left\{g_{n}\right\}_{n \in \mathbb{N}_{0}}$ of the Fourier coefficients of $g$ tends to 0 .

Before stating the main results, however, we briefly introduce some notation and terminology. As usual $\mathbb{N}$ [resp., $\mathbb{N}_{0}$ ] denotes the set of positive [resp., non-negative]

Received by the editors March 21, 1995.

1991 Mathematics Subject Classification. Primary 47A20; Secondary 47B35.

Key words and phrases. Polynomially bounded operators, similarity to contractions. 
integers, $\mathbb{C}$ is the complex plane and $\mathbb{T}$ is the unit circle in $\mathbb{C} . L^{\infty}$ is the algebra of all bounded Lebesgue-measurable functions on $\mathbb{T}$, and $H^{\infty}$ is its subalgebra consisting of those functions whose negative Fourier coefficients vanish. Similarly, $H^{2}$ is the space of square-integrable functions on $\mathbb{T}$ whose negative Fourier coefficients vanish. Finally, $\ell^{2}$ is the Hilbert space of all sequences $\left(x_{n}\right)_{n \in \mathbb{N}_{0}}$ such that $\sum_{n \in \mathbb{N}_{0}}\left|x_{n}\right|^{2}<\infty$ and $\left\|\left(x_{n}\right)\right\|_{\ell^{2}}=\left\{\sum_{n \in \mathbb{N}_{0}}\left|x_{n}\right|^{2}\right\}^{1 / 2}$. Furthermore, $z^{n}=e^{i n t}, n \in \mathbb{N}_{0}$, is the standard orthonormal basis of $H^{2}, e_{n}, n \in \mathbb{N}_{0}$, is the standard basis of $\ell^{2}\left(e_{n}\right.$ is a sequence whose only non-zero term is in the $n$th position), and there exists a Hilbert space isomorphism $V: H^{2} \rightarrow \ell^{2}$ such that $V z^{n}=e_{n}, n \in \mathbb{N}_{0}$. In what follows we shall identify $H^{2}$ with $\ell^{2}$ (and $z^{n}$ with $e_{n}$ ) without further comment. If $g \in L^{\infty}, H_{g}$ is the Hankel operator with symbol $g$, i.e., $H_{g}$ is a bounded linear operator on $H^{2}$ satisfying $H_{g}\left(z^{n}\right)=S^{* n} g$. In this situation, using the aforementioned identification between $H^{2}$ and $\ell^{2}, H_{g}$ is represented by the matrix $\left(g_{i+j}\right)_{i, j=0}^{\infty}$, where $g_{n}$ denotes the $n$th Fourier coefficient of $g$.

Lemma 1. Suppose $g \in H^{\infty}$ and the series $\sum_{n=1}^{\infty} n^{2}\left|g_{n}\right|^{2}$ does not converge. Then $T\left(H_{g}\right)$ is not similar to a contraction. In particular, this happens when $\left|g_{n}\right|=$ $(n+1)^{-3 / 2}$.

Proof. By [2, Theorem 5.3], $T(X) \in(\mathrm{SC})$ if and only if $\exists f \in H^{2}$ such that the operator $W$ defined on polynomials by

$$
W z^{n}=S^{*}\left(A_{n+1} \cdot 1-S^{* n} f\right), \quad n \in \mathbb{N}_{0},
$$

extends to a bounded linear operator on $H^{2}$. Here

$$
A_{n}=\sum_{j=0}^{n-1} S^{*(n-1-j)} X S^{j}, \quad n \in \mathbb{N} .
$$

In particular, when $X=H_{g}$, the Hankel operator with symbol $g \in L^{\infty}(\mathbb{T})$,

$$
A_{n}=\sum_{j=0}^{n-1} S^{* n-1-j} H_{g} S^{j}=\sum_{j=0}^{n-1} S^{* n-1} H_{g}=n S^{* n-1} H_{g}
$$

and therefore

$$
W z^{n}=S^{*}\left((n+1) S^{* n} H_{g} \cdot 1-S^{* n} f\right)=(n+1) S^{* n+1} H_{g} \cdot 1-S^{* n+1} f, \quad n \in \mathbb{N}_{0} .
$$

Since $H_{g} 1=g$ we have that $W z^{n}=(n+1) S^{* n+1} g-S^{* n+1} f$. Clearly, such an operator $W$, in the basis $\left\{z^{n}\right\}_{n \in \mathbb{N}_{0}}$ is represented by a matrix

$$
\left(\left\langle W z^{r}, z^{s}\right\rangle\right)_{r, s=0}^{\infty}
$$

and it is easy to see that

$$
\left\langle W z^{r}, z^{s}\right\rangle=(r+1)\left\langle g, z^{r+s+1}\right\rangle-\left\langle f, z^{r+s+1}\right\rangle=(r+1) g_{r+s+1}-f_{r+s+1} .
$$

In other words, $W$ is represented by a matrix

$$
A=\left((r+1) g_{r+s+1}-f_{r+s+1}\right)_{r, s=0}^{\infty},
$$


and for $W$ to be bounded on $H^{2}$ it is necessary that the columns of $A$ be square summable, i.e.,

$$
\sum_{r}\left|(r+1) g_{r+s+1}-f_{r+s+1}\right|^{2}<\infty, \quad \forall s \in \mathbb{N}_{0} .
$$

In particular we have that $\sum_{r}\left|(r+1) g_{r}-f_{r}\right|^{2}<\infty$, which in view of $f \in H^{2}$ implies that $\sum_{r} r^{2}\left|g_{r}\right|^{2}<\infty$, and the lemma is proved.

The main result of this paper is that $-\frac{3}{2}$ is indeed a limiting point. More precisely, we have the following

Theorem 2. Suppose that $\alpha>\frac{3}{2}$. Then there exists a function $g \in H^{\infty}$ such that $\left|g_{n}\right|=\frac{1}{(n+1)^{\alpha}}, n \in \mathbb{N}_{0}$ and such that, with $f=0$, the matrix (1) is bounded on $\ell^{2}$.

Before we can prove Theorem 2 we need to recall a few results. First, one knows that there exists a sequence $\left\{\epsilon_{n}\right\}_{n \in \mathbb{N}_{0}} \subset\{-1,1\}$ and a constant $C$ such that

$$
\sup _{z \in \mathbb{T}}\left|\sum_{n=0}^{N} \epsilon_{n} z^{n}\right| \leq C \sqrt{N}, \quad \forall N \in \mathbb{N} .
$$

Since the numbers $\epsilon_{n}$ were independently introduced by Shapiro [8] and Rudin [7], they are generally referred to as the Rudin-Shapiro coefficients.

Next, we recall that for two matrices $A=\left(a_{i j}\right)$ and $B=\left(b_{i j}\right)$ the Schur product of $A$ and $B$ is defined as $A * B=\left(a_{i j} b_{i j}\right)$. A matrix $M$ is said to be a Schur multiplier if $M * X$ defines a bounded operator on $\ell^{2}$ for every $X \in \mathcal{L}\left(\ell^{2}\right)$. The following useful test comes from [1]:

Theorem 3. Let $M$ be a matrix with $\lim _{k} m_{j k}=0=\lim _{j} m_{j k}$, and suppose that

$$
\sum_{j, k=1}^{\infty}\left|m_{j, k}-m_{j, k+1}-m_{j+1, k}+m_{j+1, k+1}\right|<\infty .
$$

Then $M$ is a Schur multiplier.

Proof of Theorem 2. Given $\alpha>\frac{3}{2}$, we define $g_{n}=\frac{\epsilon_{n}}{(n+1)^{\alpha}}, n \in \mathbb{N}_{0}$, where $\epsilon_{n}$ denotes the $n$th Rudin-Shapiro coefficient. In this situation, with $f=0$, the matrix (1) becomes

$$
A=\left((r+1) \frac{\epsilon_{r+s}}{(r+s+1)^{\alpha}}\right)_{r, s=0}^{\infty}
$$

Since $\alpha>\frac{3}{2}, \exists \beta>0$ such that $\alpha=2 \beta+\frac{3}{2}$. Using the Schur product we can rewrite $A=M * B$ where

$$
M=\left(\frac{r+1}{(r+s+1)^{1+\beta}}\right), \quad B=\left(\frac{\epsilon_{r+s}}{(r+s+1)^{\frac{1}{2}+\beta}}\right) .
$$


First we show that $B$ defines a bounded linear operator on $\ell^{2}$. We notice that $B$ is the Hankel operator corresponding to the function $h(z)=\sum_{k=0}^{\infty} \frac{\epsilon_{k}}{(k+1)^{\frac{1}{2}+\beta}} z^{k}$. Therefore it suffices to show that $h \in H^{\infty}$ because in that case $\|B\| \leq\|h\|_{\infty}$.

An easy application of the Abel summation by parts allows us to rewrite

$$
h(z)=\sum_{k=0}^{\infty}\left(\sum_{j=0}^{k} \epsilon_{j} z^{j}\right)\left[\frac{1}{(k+1)^{\frac{1}{2}+\beta}}-\frac{1}{(k+2)^{\frac{1}{2}+\beta}}\right]
$$

and therefore

$$
\|h\|_{\infty} \leq \sup _{z \in \mathbb{T}} \sum_{k=0}^{\infty}\left|\sum_{j=0}^{k} \epsilon_{j} z^{j}\right| \frac{(k+2)^{\frac{1}{2}+\beta}-(k+1)^{\frac{1}{2}+\beta}}{(k+1)^{\frac{1}{2}+\beta}(k+2)^{\frac{1}{2}+\beta}} .
$$

By the mean value theorem

$$
(k+2)^{\frac{1}{2}+\beta}-(k+1)^{\frac{1}{2}+\beta}=\left(\frac{1}{2}+\beta\right)(k+1+\theta)^{\frac{1}{2}+\beta-1}
$$

for some $0<\theta<1$. Thus, employing (2),

$$
\|h\|_{\infty} \leq \sum_{k=0}^{\infty} C \sqrt{k}\left(\frac{1}{2}+\beta\right) \frac{(k+1+\theta)^{\beta-\frac{1}{2}}}{(k+1)^{\frac{1}{2}+\beta}(k+2)^{\frac{1}{2}+\beta}}
$$

and it is easy to see that this series converges, from which it follows that $B$ is a bounded operator.

Finally, we show that $M$ is a Schur multiplier. We apply Theorem 3 with $m_{j k}=$ $\frac{j+1}{(j+k+1)^{1+\beta}}$. Clearly, both limits are 0 . Therefore, it remains to show the convergence of the double series

$$
\sum_{j, k=1}^{\infty}\left|\frac{j+1}{(j+k+1)^{1+\beta}}-\frac{j+1}{(j+k+2)^{1+\beta}}-\frac{j+2}{(j+k+2)^{1+\beta}}+\frac{j+2}{(j+k+3)^{1+\beta}}\right| .
$$

One knows that if a function $f(x, y)$ has continuous partial derivatives of the second order, then

$$
f(x, y)-f(x, y+1)-f(x+1, y)+f(x+1, y+1)=\frac{\partial^{2} f}{\partial x \partial y}(x+\theta, y+\eta),
$$

for some $0<\theta, \eta<1$. Using (3) with $f(x, y)=\frac{x+1}{(x+y+1)^{1+\beta}}$, we obtain that the series above can be written as

$$
(\beta+1) \sum_{j, k=1}^{\infty}\left|\frac{(\beta+1)(j+\theta+1)-(k+\eta)}{(j+k+\theta+\eta+1)^{\beta+3}}\right| .
$$

Since this double series is obviously convergent, the theorem is proved.

The author wishes to express his gratitude to Professor G. Bennett for many helpful discussions, to Professors C. Foias and H. Bercovici for reading the first (well, the first readable) draft of this paper, and to Indiana University for providing a pleasant and inspiring environment. 


\section{REFERENCES}

1. G. Bennett, Schur multipliers, Duke Math. J. 44 (3) (1977), 603-639. MR 58:12490

2. J. F. Carlson, D. N. Clark, C. Foias, and J. P. Williams, Projective Hilbert $\mathbb{A}(\mathbb{D})$-modules, The New York Journal of Mathematics 1 (1994), 26-38. CMP 94:15

3. C. Foias, and J. P. Williams, On a class of polynomially bounded operators, preprint.

4. P. R. Halmos, Ten problems in Hilbert space, Bull. Amer. Math. Soc. 76 (1970), 887-933. MR 42:5066

5. V. Paulsen, Every completely polynomially bounded operator is similar to a contraction, J. Funct. Anal. 55 (1984), 1-17. MR 86c:47021

6. V. Paulsen, C. Pearcy, and S. Petrović, On centered and weakly centered operators, J. Funct. Anal. 128 (1) (1995), 87-101. MR 95k:47026

7. W. Rudin, Some theorems on Fourier coefficients, Proc. Amer. Math. Soc. 10 (1959), 855-859. MR 22:6979

8. H. Shapiro, Extremal Problems for Analytic Functions and Polynomials, Dissertation, MIT, 1952.

9. B. Sz.-Nagy, Completely continuous operators with uniformly bounded iterates, Magyar Tud. Akad. Mat. Kutató Int. Köze 4 (1959), 89-93. MR 21:7436

Department of Mathematics, Indiana University, Bloomington, Indiana 47405

E-mail address: petrovic@iu-math.math.indiana.edu 